\title{
Electrical transport in single-walled carbon nanotube bundles embedded in Langmuir-Blodgett monolayers
}

\author{
V. Krstic a,b, * J. Muster ${ }^{\text {a }}$, G.S. Duesberg ${ }^{c}$, G. Philipp ${ }^{\text {a }}$, M. Burghard ${ }^{\text {a }}$, S. Roth ${ }^{\text {a }}$ \\ ${ }^{a}$ Max-Planck-Institut für Festkörperforschung, Heisenbergstr. 1, D-70569 Stuttgart, Germany \\ ${ }^{\mathrm{b}}$ Facultés Universitaires Notre-Dame de la Paix, 61, Rue de Bruxelles, B-5000, Namur, Belgium \\ ${ }^{\mathrm{c}}$ Trinity College Dublin, Physics Department, Dublin 2, Ireland
}

Received 20 August 1999; accepted 25 November 1999

\begin{abstract}
Langmuir films consisting of single-walled carbon nanotubes and surfactant molecules were deposited on substrates with lithographically defined electrode arrays. Atomic force microscopy revealed a different matrix structure of the Langmuir-Blodgett monolayers compared to monolayers on bare substrates. Electrical transport measurements were performed on individual thin bundles at room temperature and $4.2 \mathrm{~K}$. At low temperatures, the current/voltage characteristics show a non-linear dependence with step-like features, which are critically discussed in the frame of Coulomb charging theory and electron tunneling through discrete energy levels. (C) 2000 Elsevier Science S.A. All rights reserved.
\end{abstract}

Keywords: Langmuir-Blodgett techniques; Atomic force microscopy; Transport measurements; Fullerene-like materials

\section{Introduction}

Nanotubes (NTs) incorporated in (polymer) matrices attract considerable interest as composites for mechanical and optical applications. Langmuir-Blodgett (LB) films containing single-walled carbon nanotubes (SWNTs) are a suitable model system to study, e.g., the phase segregation behaviour of such materials. Moreover, monolayer transfer by the LB technique is well suited for covering an electrode array with just a few single NTs or NT bundles, which allows electrical measurements on individual objects.

Recent electrical transport investigations on adsorbed individual SWNTs [1] and individual bundles of SWNTs [2] provided evidence for electron energy quantization due to the finite tube length. Theoretical [3] and experimental [4] studies suggest that tube bending introduces local electronic defects that impede the electron propagation along the NT.

In the present study, LB monolayers, consisting of NTs homogeneously distributed in a matrix of amphiphilic

\footnotetext{
* Corresponding author. Tel.: +49-711-689-1343; fax: +49-711-6891010.

E-mail address: krstic@klizik.mpi-stuttgart.mpg.de (V. Krstic).
}

molecules, were deposited on a substrate with electronlithographically defined electrode arrays. One goal was to elucidate the matrix structure and its influence on the electrical transport through the embedded SWNTs. The information obtained from the two-terminal current/voltage $(I / V)$ characteristics of the SWNT bundles is critically discussed in terms of Coulomb charging and tunnelling through discrete energy levels.

\section{Experimental}

The electrode arrays were produced by conventional electron beam lithography (EBL) using a modified SEM (Hitachi 2300) and a two-layer poly(methyl methacrylate) (PMMA) resist system. $\mathrm{Au} / \mathrm{Pd}(60$ at.\% / 40 at.\%) electrodes $14 \mathrm{~nm}$ in height, approximately $100 \mathrm{~nm}$ in width and $2 \mu \mathrm{m}$ in length and spaced by about $100 \mathrm{~nm}$ were defined on an Si wafer with a $1 \mu \mathrm{m}$ thick thermally grown $\mathrm{SiO}_{2}$ layer.

In order to remove residual PMMA resist, the substrate was irradiated for $1 \mathrm{~h}$ with monochromatic UV light $(\lambda=254 \mathrm{~nm})$ at a distance of about $1 \mathrm{~cm}$ from the UV source of $6 \mathrm{~W}$ power. Then the substrate was successively rinsed with acetone and isopropanol. Finally, it was 
silanized in hexamethyldisilazane/chloroform (1 vol:1 vol) for $12 \mathrm{~h}$ at room temperature.

SWNT raw material produced by the arc discharge method was dispersed in an aqueous lithium dodecyl sulfate (LDS) solution with the aid of ultrasonic agitation. Then the dispersion was purified and spread onto an aqueous subphase $\left(T=21^{\circ} \mathrm{C}\right)$ containing poly(allylamine hydrochloride) as described recently [5].

For LB film preparation, a commercially available trough with two movable barriers was used. After spreading of the purified dispersion, the Langmuir film was compressed at a rate of $10 \mathrm{~mm} / \mathrm{min}$ and kept at a surface pressure of $\pi=14 \mathrm{mN} / \mathrm{m}$. Film stabilization was observed after 5-10 min.

Subsequently, the Langmuir film was deposited horizontally according to the Schaefer method [6] by lowering the substrate with a speed of $1 \mathrm{~mm} / \mathrm{min}$ and removing it after a period of $1 \mathrm{~min}$ with a speed of $0.5 \mathrm{~mm} / \mathrm{min}$. The transfer ratio was found to be close to 2 , which is explained by restructuring of the surfactant matrix [5]. Deposited monolayers were investigated by atomic force microscopy (AFM) in Tapping Mode (Digital Instruments, Nanoscope IIIa). Electrical measurements were performed under $\mathrm{He}$ atmosphere at room and liquid helium temperature using a standard DC set-up applying voltage and measuring current.

\section{Results and discussion}

In Fig. 1, an AFM image of an LB monolayer on an electrode array is shown. The LDS matrix appears to be flat, in contrast to monolayers deposited on a bare $\mathrm{SiO}_{2}$

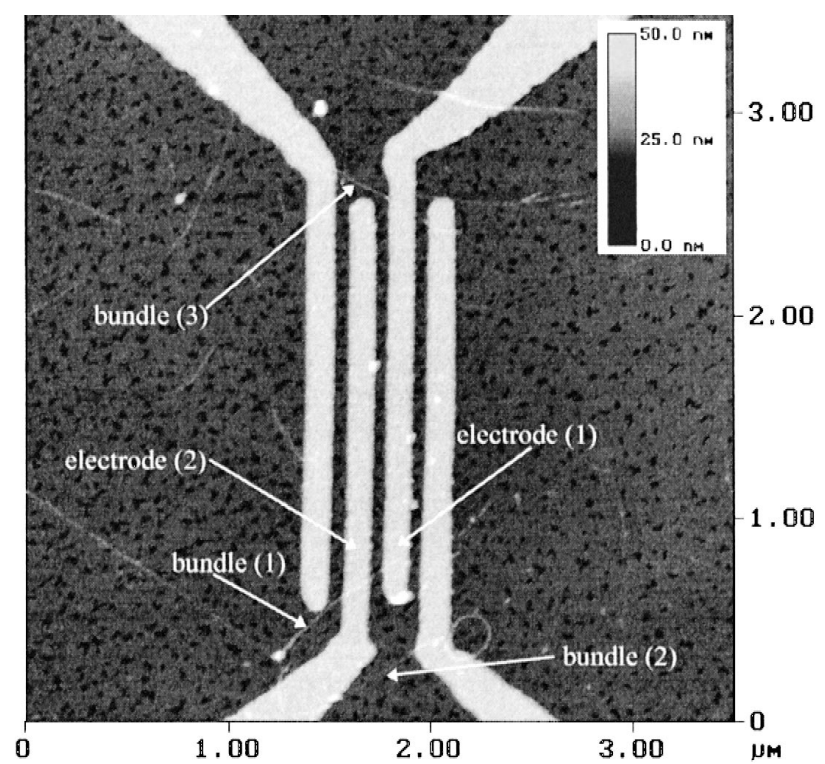

Fig. 1. AFM image of an LB monolayer consisting of SWNTs embedded in the surfactant matrix deposited on electrodes. The electrically investigated bundles are marked with arrows.
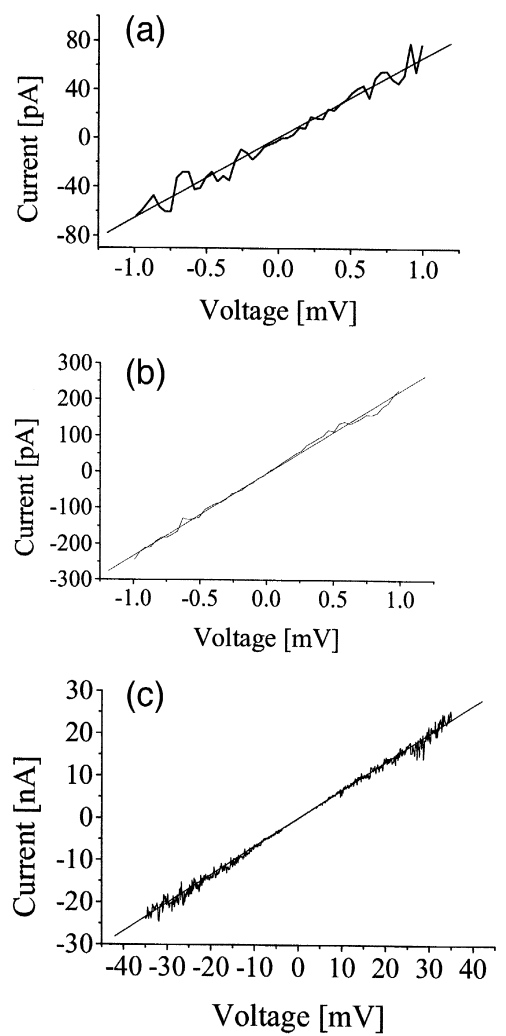

Fig. 2. The $I / V$ characteristics at room temperature of the bundles (1), (2) and (3) are shown in (a), (b) and (c), respectively. Note the current scale in (b), in contrast to (a) and (c). The solid lines are linear fits.

surface [5]. The different matrix structure probably results from a modification of the substrate surface by the EBL procedure. The matrix is not fully continuous, i.e., it exhibits holes (black spots in the AFM image). This allows one to determine the height of the matrix, which was found from the AFM section profiles to be about $2.5 \mathrm{~nm}$.

The SWNTs and SWNT bundles are well separated and homogeneously distributed. Moreover, in contrast to LB films of other matrix-embedded macromolecules [7], the tubes do not agglomerate into domains. Although the matrix is not fully continuous, the tubes are almost completely enclosed by the surfactant molecules.

In our experiments, the pure surfactant matrix exhibited a resistance larger than $10^{12} \Omega$. The room temperature $I / V$ characteristic of the bundles (1), (2) and (3) is shown in Fig. 2a, b and c, respectively. In all three cases, the room temperature characteristic shows a linear dependence. The significant current fluctuations are attributed to thermally induced instabilities of the electrical contact between bundle and electrode. From the slope of the linearly fitted lines (solid), one obtains a resistance of $(14.6 \pm 0.4) \mathrm{M} \Omega$ for bundle (1), $(1.8 \pm 0.1) \mathrm{M} \Omega$ for bundle (2) and (5 \pm 0.2$) \mathrm{M} \Omega$ for bundle (3). These values are within the range observed for SWNTs adsorbed on prefabricated electrodes $[1,8]$. It is, therefore, concluded that the surfactant matrix does not form a closed insulating layer between bundle and electrode, and that only a native 

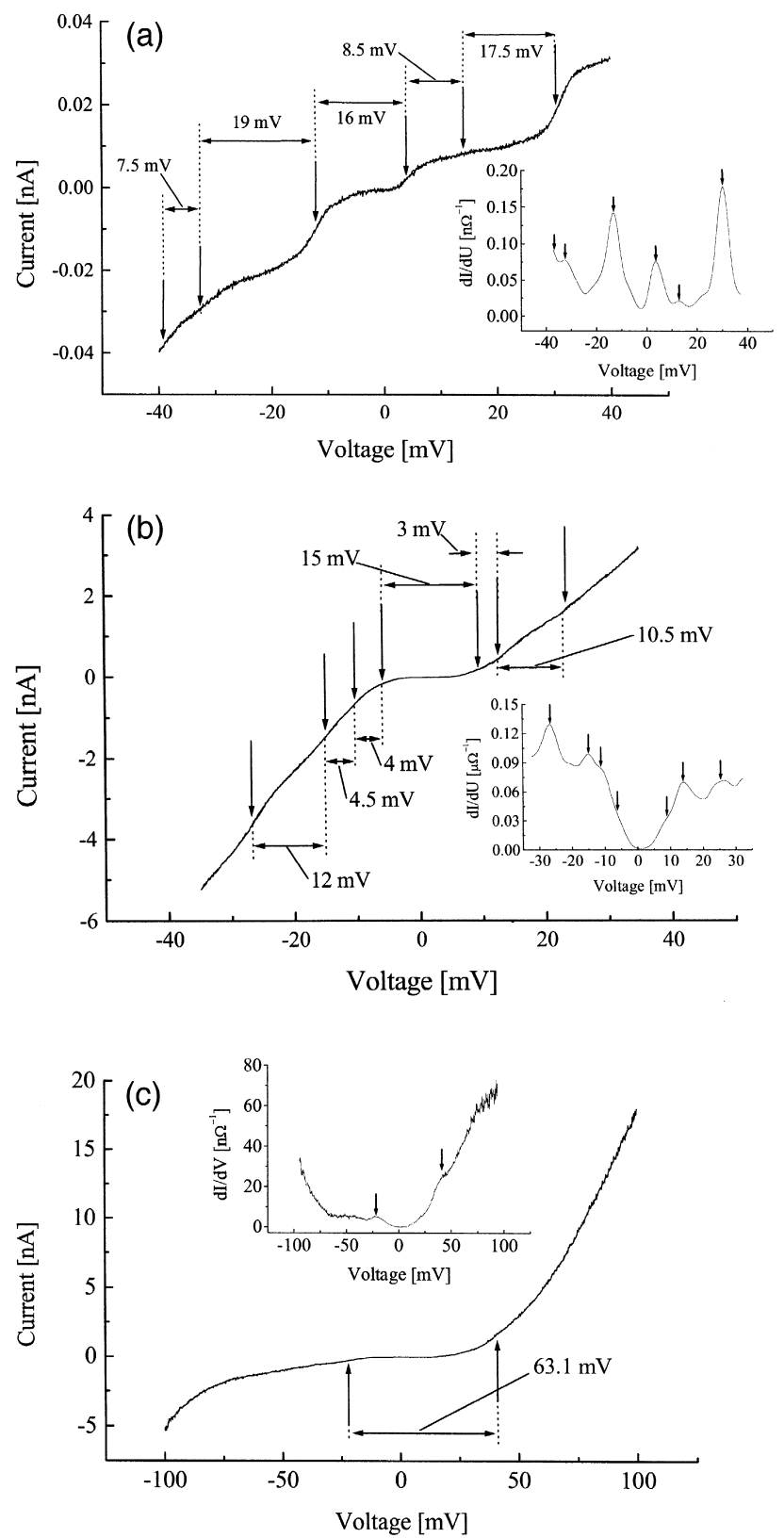

Fig. 3. (a) The $I / V$ characteristics of bundle (1) at $4.2 \mathrm{~K}$. Note that bundle (1) is the only one that bridges neighbouring electrodes and it has the largest ratio between the length of the full bundle and of the part between the electrodes. The dimensions of bundle (1) are: $(2.5 \pm 0.5) \mathrm{nm}$ in diameter, $(830 \pm 10) \mathrm{nm}$ entire bundle length and $(90 \pm 5) \mathrm{nm}$ segment length between electrodes. (b) The $I / V$ characteristic at $4.2 \mathrm{~K}$ of bundle (2). (c) The $I / V$ characteristic of bundle (3) shows two weakly pronounced steps. The insets show the first derivative (Fourier-filtered) of the curves. Arrows mark the step positions.

hydrocarbon adsorbate layer [9] exists on the $\mathrm{Au} / \mathrm{Pd}$ electrodes.

At liquid helium temperature, the thermally induced fluctuations are suppressed. Additionally, the $I / V$ characteristic of bundle (1) (Fig. 3a) exhibits step-like features. The $I / V$ curve of bundle (2) also shows steps, although less pronounced (Fig. 3b). Only two weak steps are observed in the case of bundle (3) (Fig. 3c). The insets show the first derivative with the arrows marking the step positions.

One might be tempted to interpret the current steps in the $I / V$ characteristic of the bundles in terms of Coulomb charging and single electron tunnelling through discrete energy levels and to evaluate whether the SWNT bundles are segmented due to its bending over the electrodes.

In the following, the limits of such an approach are discussed, as exemplified for the specific case of bundle (1). It should be noted that the same conclusions hold for bundle (2), whereas the number of steps in the $I / V$ characteristic of bundle (3) is not sufficient for an analysis within this framework.

In the frame of Coulomb charging theory, the SWNT bundle and the surrounding electrodes can be regarded as a nanoscale "island" (or dot) with contact resistances much larger than $h / \mathrm{e}^{2} \approx 25.8 \mathrm{k} \Omega$. Such an island exhibits current steps in the $I / V$ characteristic with spacings determined by $2 E_{\mathrm{C}}$ ( $E_{\mathrm{C}}$ : charging energy of the island), or the discrete level spacing $\Delta E$, or both [10]. $E_{\mathrm{C}}$ is obtained by $E_{\mathrm{C}}=(1 / 2) \mathrm{e}^{2} / C_{\Sigma}$

where $C_{\Sigma}$ is the total capacitance of the island, given by the sum over capacitances $C_{i}$ with $C_{i}$ being the capacitance of the bundle to the electrode $i$ [11].

For a first approximation of $E_{\mathrm{C}}$ for the SWNT bundle $[1,4]$, its self-capacitance $C_{\text {self }}$, which depends on its diameter $d$ and its electrically active length $L[1]$, is set equal to $C_{\Sigma}$. The self-capacitance of the entire (upper index (e)) SWNT bundle and the bundle segment (upper index (s)) between the electrodes, $C_{\text {self }}^{(\mathrm{e})}$ and $C_{\text {self }}^{(\mathrm{s})}$, can be estimated assuming the bundle to be of ellipsoidal shape with $d \ll L$. Thus,

$C_{\Sigma} \equiv C_{\text {self }}^{(j)} \approx 2 \pi \varepsilon_{0} \varepsilon_{\mathrm{r}} L\{\ln (2 L / d)\}^{-1} \quad(j=\mathrm{e}, \mathrm{s})$

with the vacuum permittivity $\varepsilon_{0}$ and the permittivity of the carbon bundle $\varepsilon_{\mathrm{r}} \approx 3$ (as for graphite) [12]. The calculated values of $C_{\text {self }}^{(\mathrm{e})}$ and $C_{\text {self }}^{(\mathrm{s})}$ are presented in Table 1 . The spacing $\Delta E$ of the discrete energy levels, which is due to the finite length $L$ of a bundle, is approximately given by $0.5 / L \mathrm{eV}$, where $L$ is given in $\mathrm{nm}$ [13].

To convert the voltage scale in Fig. 3a into an energy scale, the capacitive coupling factor $\alpha$ between bundle and biased electrode is needed [11]. In a recent article by Bockrath et al. [14], a symmetric coupling, i.e., $\alpha=1 / 2$, was assumed. Using $\alpha=1 / 2$, the maximum step separa-

Table 1

Self-capacitances, $C_{1}$ and $C_{2}$

Estimated values for $C_{\Sigma}^{(\mathrm{s})}, C_{\Sigma}^{(\mathrm{e})}, C_{1}$ and $C_{2} . L_{1}=(90 \pm 5) \mathrm{nm}, L_{2}=(160$ $\pm 10) \mathrm{nm}$. The dimensions of bundle (1) are given in Fig. 3.

\begin{tabular}{llll}
\hline$C_{\text {self }}^{(\mathrm{s})}[\mathrm{aF}]$ & $C_{\text {self }}^{(\mathrm{e})}[\mathrm{aF}]$ & $C_{1}[\mathrm{aF}]$ & $C_{2}[\mathrm{aF}]$ \\
\hline $3.5 \pm 0.2$ & $21.3 \pm 0.7$ & $3.3 \pm 0.3$ & $5.9 \pm 0.5$ \\
\hline
\end{tabular}


tion $\Delta V$ of $19 \mathrm{mV}$ (Fig. 3) is converted to e $\Delta V \alpha=9.5$ $\mathrm{meV}$. This value is quite close to but yet larger than $2 E_{\mathrm{C}}^{(\mathrm{e})}=(7.5 \pm 0.2) \mathrm{meV}$ calculated by Eqs. (1) and (2). On the other hand, $2 E_{\mathrm{C}}^{(\mathrm{s})}=(42.2 \pm 2.6 \mathrm{meV})$ is too large, and $\Delta E^{(\mathrm{e})}=(0.6 \pm 0.01) \mathrm{meV}$ and $\Delta E^{(\mathrm{s})}=(5.5 \pm 0.3) \mathrm{meV}$ are both too small.

At this point, it has to be noted that $C_{\Sigma}$ is close to the self-capacitance $C_{\text {self }}$ of the nanoscale object between the electrodes only if the $C_{i} \mathrm{~s}$ and the sum of the $C_{i}$ s are negligible compared to the magnitude of $C_{\text {self }}$. If not, the actual value of $C_{\Sigma}$ is larger than $C_{\text {self }}$.

Considering the size and the geometrical arrangement of the electrodes (Fig. 1), $C_{1}$ and $C_{2}$ can be estimated using a plate-capacitor model. Towards this end, the spacing $S$ of the plates is approximated by the sum of the typical thickness $h$ of the hydrocarbon adsorbate layer ( $\approx 0.5 \mathrm{~nm}$ for flat lying chains) and half of the diameter of the bundle $d / 2$. The $C_{i}$ s are then given by

$C_{i}=\varepsilon_{0} \varepsilon_{\mathrm{r}} d L_{i} / S \quad(i=1,2), \quad S=h+(d / 2)$

with $L_{i}$ being the length of the bundle over electrode $i$ and $\varepsilon_{\mathrm{r}} \approx 3$ the permittivity of the hydrocarbon layer [15]. The calculated capacitances of the two bundle-electrode contacts $C_{1}$ and $C_{2}$ are given in Table 1 .

As an important result, the estimated $C_{1}$ and $C_{2}$ are of the same order of magnitude as $C_{\text {self }}^{(\mathrm{s})}$. The sum of $C_{1}$ and $C_{2}$ is almost the half of $C_{\text {self }}^{(\mathrm{e})}$. This comparison shows that the influence of the electrodes on the electron system of the SWNT bundle, due to the screened electron-electron interaction in the region of the electrodes [16], is substantial and consequently $C_{\Sigma}$ differs considerably from $C_{\text {self }}^{(\mathrm{e})}$ or $C_{\text {self }}^{(\mathrm{s})}$.

Using a value $C_{\Sigma}>C_{\text {self }}^{(\mathrm{s})}$, values of $2 E_{\mathrm{C}}^{(\mathrm{s})}$ are obtained which approach the observed maximum step separation of e $\Delta V \alpha=9.5 \mathrm{meV}$. In contrast, $2 E_{\mathrm{C}}^{(\mathrm{e})}$ deviates even more. Therefore, the electrically active length seems to be close to that of the bundle segment.

However, in the case of an asymmetric capacitive coupling, $\alpha$ would be either larger or smaller than $1 / 2$. For $\alpha<1 / 2$, the entire bundle length would be assumed to be electrically active. If $\alpha>1 / 2$, again the bundle segment would be interpreted as the electrically active length. It follows from these considerations that our experimental data cannot be used to decide whether the SWNT bundle is segmented due to tube bending or not.

The above discussion demonstrates that any interpretation of the experimental data should take into account a number of crucial points. It is the total capacitance of the island $C_{\Sigma}$ that has to be used to evaluate the charging energy $E_{\mathrm{C}}$. The self-capacitance of the SWNT bundle $C_{\text {self }}$ should be approximated by $C_{\Sigma}$, only if the $C_{i}$ s are small compared to $C_{\text {self }}$. Otherwise, equating $C_{\Sigma}$ with $C_{\text {self }}$ would lead to misinterpretation of experimental data. Moreover, the capacitive couplings of the SWNTs to the electrodes are needed to convert the voltage scale of the $I / V$ characteristics into an energy scale. The knowledge of these couplings would allow a correct comparison between observed step separations and estimated values of $E_{\mathrm{C}}$.

\section{Conclusion}

Langmuir films consisting of SWNTs and surfactant molecules were successfully deposited on electrode arrays. The surfactant matrix exhibits a different structure than on bare $\mathrm{Si} / \mathrm{SiO}_{2}$ wafers. The embedded SWNTs and SWNT bundles are homogeneously distributed and no agglomeration into domains could be observed. The room temperature resistance in the $\mathrm{M} \Omega$ range excludes a fully insulating surfactant layer between bundles and electrodes. At a temperature of $4.2 \mathrm{~K}$, steps are observed in the $I / V$ characteristic. Their interpretation is difficult due to the unknown capacitive coupling factors of the electrodes to the bundle. These factors could be obtained by gate-dependent measurements.

It has to be noted, however, that the nanotube is not an isolated object. The presence and geometry of the electrodes strongly influence the total capacitance of the nanoscale object and thus its transport properties. For the present contact geometry, this means that the total capacitance $C_{\Sigma}$ can easily exceed the self-capacitance $C_{\text {self }}$ of the SWNT bundle. It was discussed that $C_{1}$ and $C_{2}$ are of the same order of magnitude as $C_{\mathrm{self}}^{(j)}(j=\mathrm{e}, \mathrm{s})$. Therefore, it is impossible to distinguish whether the SWNT bundle is segmented due to tube bending or not.

\section{Acknowledgements}

We would like to thank P. Bernier and C. Journet for providing SWNT raw material. In addition, we are grateful to $\mathrm{J}$. Weis for helpful discussions.

\section{References}

[1] S.J. Tans, M.H. Devoret, H. Dai, A. Thess, R.E. Smalley, L.J. Geerligs, C. Dekker, Nature 386 (1997) 474.

[2] M. Bockrath, D.H. Cobden, P.L. McEuen, N.G. Chopra, A. Zettl, A. Thess, R.E. Smalley, Science 275 (1992) 1922.

[3] A. Rochefort, P. Avouris, F. Lesage, D.R. Salahub, Phys. Rev. B 60 (1999) 13824.

[4] A. Bezryadin, A.R.M. Verschueren, S.J. Tans, C. Dekker, Phys. Rev. B 80 (1998) 4036.

[5] V. Krstic, G.S. Duesberg, J. Muster, M. Burghard, S. Roth, Chem. Mater. 10 (1998) 2338.

[6] A. Ulman, An Introduction to Ultrathin Organic Films - from Langmuir-Blodgett to Self-Assembly, Academic Press, San Diego, 1991.

[7] J. Wu, G. Lieser, G. Wegner, Adv. Mater. 8 (1996) 151.

[8] S.J. Tans, A.R.M. Verschueren, C. Dekker, Nature 393 (1998) 49.

[9] M.C. Petty, W.A. Barlow, in: Langmuir-Blodgett Films Plenum, New York, 1990, pp. 115-118, Chap. 3.

[10] A.N. Korotkov, in: Molecular Electronics Blackwell, Oxford, UK, 1997, pp. 178-184, Chap. 4. 
[11] J. Weis, R.J. Haug, K.v. Klitzing, K. Ploog, Phys. Rev. B 46 (1992) 12837.

[12] Gmelins Handbuch d. Anorg. Chemie B2, 8th edn., Verlag Chemie, Weinheim, 1968.

[13] D.H. Cobden, M. Bockrath, N.G. Chopra, A. Zettl, P.L. McEuen, A. Rinzler, A. Thess, R.E. Smalley, Physica B 132 (1998) 249-251.
[14] M. Bockrath, D.H. Cobden, J. Lu, A. Rinzler, R.E. Smalley, L. Balents, P.L. McEuen, Nature 397 (1999) 598-601.

[15] CRC Handbook of Chemistry and Physics, 64th edn., CRC Press, Boca Raton, 1983-1984, pp. 49-54, Chap. E.

[16] L.D. Hallam, J. Weis, P.A. Maksym, Phys. Rev. B 53 (1996) 1452. 